Subscriber access provided by Lib4RI - Library for Eawag, Empa, PSI \& WSL

\title{
Article
}

\section{Impact of in-sewer degradation of pharmaceutical and personal care products (PPCPs) population markers on a population model}

Jake William O'Brien, Andrew Phillip William Banks, Andrew Joseph Novic, Jochen F. Mueller, Guangming Jiang, Christoph Ort, Geoff Eaglesham, Zhiguo Yuan, and Phong Thai

Environ. Sci. Technol., Just Accepted Manuscript •DOI: 10.1021/acs.est.6b02755 • Publication Date (Web): 28 Feb 2017

Downloaded from http://pubs.acs.org on March 7, 2017

\section{Just Accepted}

"Just Accepted" manuscripts have been peer-reviewed and accepted for publication. They are posted online prior to technical editing, formatting for publication and author proofing. The American Chemical Society provides "Just Accepted" as a free service to the research community to expedite the dissemination of scientific material as soon as possible after acceptance. "Just Accepted" manuscripts appear in full in PDF format accompanied by an HTML abstract. "Just Accepted" manuscripts have been fully peer reviewed, but should not be considered the official version of record. They are accessible to all readers and citable by the Digital Object Identifier (DOI $($ ). "Just Accepted" is an optional service offered to authors. Therefore, the "Just Accepted" Web site may not include all articles that will be published in the journal. After a manuscript is technically edited and formatted, it will be removed from the "Just Accepted" Web site and published as an ASAP article. Note that technical editing may introduce minor changes to the manuscript text and/or graphics which could affect content, and all legal disclaimers and ethical guidelines that apply to the journal pertain. ACS cannot be held responsible for errors or consequences arising from the use of information contained in these "Just Accepted" manuscripts. 
1 Title: Impact of in-sewer degradation of pharmaceutical and personal care products (PPCPs)

2 population markers on a population model

3 Authors:

4 Jake William O'Brien*a

5 Andrew Phillip William Banks ${ }^{\mathrm{a}}$

6 Andrew Joseph Novic ${ }^{a}$

7 Jochen F. Mueller ${ }^{\mathrm{a}}$

8 Guangming Jiang ${ }^{\mathrm{b}}$

9 Christoph Ort ${ }^{\mathrm{c}}$

10 Geoff Eaglesham $^{a}$

11 Zhiguo Yuan ${ }^{b}$

12 Phong K. Thai ${ }^{\mathrm{a}, \mathrm{d}}$

${ }^{a}$ Queensland Alliance for Environmental Health Science (QAEHS), The University of Queensland, 39 Kessels Road, Coopers Plains, QLD 4108, Australia

${ }^{b}$ Advanced Water Management Centre (AWMC), The University of Queensland, St. Lucia, QLD 4072, 17 Australia

${ }^{\mathrm{C} E a w a g}$, Swiss Federal Institute of Aquatic Science and Technology, CH 8600 Dübendorf, Switzerland ${ }^{\mathrm{d} I n t e r n a t i o n a l ~ L a b o r a t o r y ~ f o r ~ A i r ~ Q u a l i t y ~ a n d ~ H e a l t h, ~ Q u e e n s l a n d ~ U n i v e r s i t y ~ o f ~ T e c h n o l o g y, ~ B r i s b a n e, ~}$ 20 QLD 4001, Australia

$21 *$ j.obrien2@uq.edu.au

*Tel. +61 (0)7 3274 9009, Fax +61 (0)732749003

tQueensland Alliance for Environmental Health Sciences incorporates the former National Research Centre for Environmental Toxicology (Entox).

Abstract

A key uncertainty of wastewater-based epidemiology is the size of the population which contributed to a given wastewater sample. We previously developed and validated a Bayesian inference model to estimate population size based on 14 population markers which: (1) are easily measured and (2) have mass loads which correlate with population size. However, the potential uncertainty of the model prediction due to in-sewer degradation of these markers was not evaluated. In this study we addressed this gap by testing their stability under sewer conditions and assessed whether degradation impacts the model estimates. Five markers which formed the core of our model, were 
35 population markers in the model did not decrease the precision of the population estimates

36 providing that stable markers such as acesulfame remained in the model. However, to achieve the

37 minimum uncertainty in population estimates, we propose that the core markers to be included in

38 population models for other sites should meet two additional criteria: (3) negligible degradation in

39 wastewater to ensure the stability of chemicals during collection; and $(4)<10 \%$ in-sewer degradation

40 could occur during the mean residence time of the sewer network.

41

42

43

44

45

46

47

48

49

50

51

52

53

\section{Introduction:}

Wastewater-based epidemiology (WBE) has become a useful approach for monitoring of drug use at the population level ${ }^{1-8}$. Challenges remain to improve the accuracy of the approach and a key uncertainty associated with WBE relates to the population that has contributed to a given wastewater sample ${ }^{1}$ which is essential for the normalisation of the estimates. Normalised data is important to ensure that WBE is comparable across cities and even countries. A number of proxies have previously been proposed to address the population uncertainty. These include nutrients such as $\mathrm{N}$ and $\mathrm{P}$, biological oxygen demand (BOD) and chemical oxygen demand (COD) ${ }^{9,10}$, creatinine ${ }^{11}$, caffeine and nicotine ${ }^{12}$, as well as selected pharmaceuticals and personal care products (PPCPs) and an artificial sweetener ${ }^{3}$. But none of the proposed population markers were calibrated against accurate population counts and thus were not validated yet. In our previous paper, a Bayesian inference model was developed to estimate de facto population size using 14 anthropogenic markers (Table 1) ${ }^{13}$ using samples from 10 wastewater treatment plants (WWTPs) collected during the 2011 Australian Census. We subsequently applied this model successfully to support a study on daily drug use monitoring in a catchment in South East Queensland, Australia ${ }^{2}$. However, we recognised that some chemicals may degrade in the sewer ${ }^{14}$. Therefore, it is important to investigate whether degradation during sewer passage contributes to the uncertainty of the population estimation model. 
61 In-sewer degradation is a result of both hydrochemical and biotransformation processes in the 62 sewer and as such is dependent on the chemistry (e.g. metals, micropollutants, nutrients, $\mathrm{pH}$ ) of the 63 wastewater, the bioactivity in the sewer and residence time of the wastewater in the sewer ${ }^{15,16}$. In 64 real sewers the chemistry, bioactivity and residence times are dependent on the flow and 65 composition of water entering from the catchment at the time ${ }^{16}$. Additionally, sewers are complex systems comprising of a multitude of pipes ranging from small, pressurised pipes called rising mains (RM) to large partially filled gravity fed pipes called gravity sewers (GS). As such the surface area, which biofilm can grow on is, dependent on both the size of the pipe and how full the pipe remains. The ratio of area of biofilm versus volume of wastewater is thus termed the A/V ratio. Sewer networks comprise of sections ranging from low $A / V$ to high $A / V$ with differing residence times in each. A major limitation of in-sewer chemical degradation monitoring is that the observed degradation rates need to be statistically higher than the uncertainty of the chemical analysis and thus high $\mathrm{A} / \mathrm{V}$ and residence times may be required. Using laboratory-scale sewer reactors overcomes this limitation as both the $\mathrm{A} / \mathrm{V}$ and residence time can be controlled ${ }^{14,17}$.

The aim of this study is to 1 ) assess the stability of the 14 population markers proposed in O'Brien et al. ${ }^{13}$ under sewer conditions and 2) assess whether potential degradation of these population markers in the sewer impacts the population model. From this we propose a protocol to assess whether a chemical can be used as a population marker.

\subsection{Chemicals and reagents}

Analytical grade acetic acid was purchased from Sigma Aldrich (Castle Hill, Australia). Analytical grade hydrochloric acid 32\% was purchased from Univar (Ingleburn, Australia). Water was purified through a MilliQ system (Millipore, $0.22 \mu \mathrm{m}$ filtered, $18.2 \mathrm{~m} \Omega \mathrm{cm}^{-1}$ ). High purity PPCP native and 
labelled analytical standards were purchased from various suppliers as outlined in the SI. Calibration standards were prepared in MilliQ water. Liquid chromatography grade methanol was purchased from Merck (Darmstadt, Germany). Mobile phases were filtered using Sartorius Stedim $0.45 \mu \mathrm{m}$ RC filters (Goettingen, Germany).

\subsection{Laboratory-scale sewer reactors}

Laboratory-scale sewer reactors, with high $\mathrm{A} / \mathrm{V}\left(70.9 \mathrm{~m}^{2} / \mathrm{m}^{3}\right)$ which is similar to $\mathrm{A} / \mathrm{V}$ ratios of small pipes, were used to investigate the biotransformation and degradation of the population markers. These reactors have been described elsewhere ${ }^{14,18,19}$. Briefly, the system comprised of laboratoryscale RM, GS and a control reactor (CR). Earlier studies using these reactors have found them suitable for studies regarding in-sewer processes, the control of sewer biofilm activities and the insewer degradation of chemicals ${ }^{14,17-22}$. The sewer reactors are fed with domestic wastewater collected on a weekly basis from a local pumping station (Indooroopilly, Brisbane) which is typically at $\mathrm{pH} 7.5$ with sulphide concentrations $<3 \mathrm{mg} \mathrm{S} / \mathrm{L}$, sulfate between 10 and $25 \mathrm{mg} \mathrm{S} / \mathrm{L}$, total COD between 450 and $600 \mathrm{mg} / \mathrm{L}$, soluble COD between 260 and $450 \mathrm{mg} / \mathrm{L}$ which contains volatile fatty acids between 50 to $120 \mathrm{mg} \mathrm{COD} / \mathrm{L}^{19}$. The collected wastewater is stored at $4{ }^{\circ} \mathrm{C}$ and warmed to $\sim 20$ ${ }^{\circ} \mathrm{C}$ prior to feeding into the reactors. Feeding events occur at 6 hour intervals to mimic the typical hydraulic retention time of a real sewer ${ }^{23}$ and last approximately 2 minutes during which time one reactor volume $(0.75 \mathrm{~L})$ of wastewater is delivered to the reactor. Magnetic stirrers set to $250 \mathrm{rpm}$ (Heidolph MR3000) homogenise the wastewater in the reactors. Further description of the reactors can be found in Thai et al. ${ }^{14}$.

\subsection{Batch tests for the degradation of PPCPs in sewer reactors}

To investigate the degradation of the PPCPs under different sewer conditions, batch tests were conducted in triplicate. To meet OECD guideline No. 314, wastewater from the sampling pump station was warmed to $20^{\circ} \mathrm{C}$ however no $\mathrm{pH}$ adjustment was necessary ${ }^{24}$. These conditions are comparable with other studies on the stability of chemicals under sewer conditions ${ }^{19,25-27}$. 
111 Preliminary analysis of the wastewater was conducted to determine the spiking amount. Only

112 furosemide, iopromide and norfloxacin required spiking for degradation to be measured accurately

113 to give a spiked concentration of $5-10 \mu \mathrm{g} \cdot \mathrm{L}^{-1}$ above concentrations already present in the wastewater

114 but within the range usually found in previous wastewater studies. For this purpose, a working

115 solution of PPCP standards from $5-10 \mu \mathrm{g} \cdot \mathrm{mL}^{-1}$ was prepared in 50:50 methanol/deionised water and

116 spiked in to the wastewater. Other chemicals can be tested with the residues already available in the

117 wastewater matrix. The wastewater was then pumped using a peristaltic pump (Masterflex 7520-47)

118 at a flow rate of $0.375 \mathrm{~L} / \mathrm{min}$ into the RM and GS reactors ensuring that the wastewater in each

119 reactor was entirely replaced with fresh wastewater. Mixing occurred continuously for the duration

120 of the batch tests using magnetic stirrers set to $250 \mathrm{rpm}$ (Heidolph MR3000). This not only improved

121 homogeneity of the wastewater but also enhanced surface aeration (dissolved oxygen of $\sim 0.5 \mathrm{mg} / \mathrm{L}$ )

122 producing both aerobic and anaerobic conditions in the GS reactor. The CR was basically the same as

123 the GS except that the walls are regularly cleaned hence preventing the growth of biofilms, however,

124 mixing still occurred which enhanced the aerobic conditions for the $C R$ ( $D O=0.5 \mathrm{mg} / \mathrm{L}$ ). Wastewater

125 samples were taken at $0,0.25,0.5,1,2,3,6,9$ and 12 hours after the feeding event. Samples were

126 immediately filtered using $0.20 \mu \mathrm{m}$ PTFE syringe filters (Phenomenex, Australia) into $2 \mathrm{~mL}$ vials and

$12715 \mu \mathrm{L}$ of $2 \mathrm{M} \mathrm{HCl}$ was added to each sample to acidify the sample to $\sim \mathrm{pH} 2$ to reduce the biological

128 activity ${ }^{19}$ and then samples were frozen at $-20^{\circ} \mathrm{C}$ prior to analysis.

130 A slightly modified version of the chemical analysis outlined in $\mathrm{O}^{\prime}$ Brien et al ${ }^{13}$ was performed using a

131 Sciex QTrap 6500 (Sciex, Concord, Ontario, Canada) with electrospray ionization (ESI) interfaces

132 coupled to Shimadzu Nexera HPLC systems (Shimadzu Corp., Kyoto, Japan). Separation was achieved

133 on a Kinetix Biphenyl column (2.6 $\mu \mathrm{m}, 100 \AA ̊$, LC Column $50 \mathrm{~mm} \times 2.1 \mathrm{~mm}$, Phenomenex $)$ using a

134 mobile phase gradient of 1 to $95 \%$ methanol with $0.1 \%$ acetic acid. The QTrap 6500 was operated in

135 scheduled multiple reaction monitoring (sMRM) mode with optimised parameters (SI Table 1). Data 
136 acquisition was performed using the Sciex software package Analyst Software 1.6 and quantification

137 was performed using MultiQuant 3.0. Quantification was done using relative response factors of

138 mass labelled internal standards.

139 For dissolved sulphide, samples were analysed within $24 \mathrm{~h}$ of sampling using an ion chromatograph

140 with an UV and conductivity detector (Dionex ICS-2000). For methane analysis, BD vacutainer tubes

141 were allowed to reach gas/liquid equilibrium overnight. Methane in the gas phase was measured by

142 gas chromatography (Shimadzu GC-9A) equipped with a flame ionization detector. Concentrations of

143 methane in sewage were calculated using mass balance and Henry's law ${ }^{28}$.

$144 \quad 3 \quad$ Results and Discussion:

\subsection{Biological activities in sewer reactors}

The biological activity indicated by methane and sulphide gas production of the RM and GS reactors

used in this experiment has previously been found to represent the biological activity and associated

degradation of chemicals within actual sewers ${ }^{14}$. The control reactor showed no significant methane or sulphide gas production as it doesn't contain the sewer biofilms compartment as seen before ${ }^{19}$. The RM reactor produced sulphide and methane indicating that it was under anaerobic conditions. Activities of sulphate-reducing bacteria and methanogenic archaea in the RM reactor were measured at $7.21 \pm 0.74 \mathrm{mg} \mathrm{S} / \mathrm{L}-\mathrm{h}$ and $29.73 \pm 0.63 \mathrm{mg} \mathrm{COD} / \mathrm{L}-\mathrm{h}$ respectively which is similar to previously reported values for both real and laboratory-scale sewers ${ }^{19,22,28,29}$. Dissolved oxygen in the GS reactor was measured below $0.33 \mathrm{mg} / \mathrm{L}$ despite continuous aeration which indicates aerobic activity consuming oxygen. It is also expected that anaerobic conditions may be present in the bottom of the reactor where oxygen could not reach. This is supported by the low reduction of sulphate (4.21 $\pm 0.77 \mathrm{mg} \mathrm{S} / \mathrm{L}-\mathrm{h})$ and the low production of methane (14.8 $\pm 2.31 \mathrm{mg}$ COD/L-h). 
159 Figure 1 shows the concentration profile of chemicals of interest in the system over time normalised

160 to the concentration at the start of the experiment expressed in percent (i.e. $C_{t} / C_{0} \times 100$ ). To

161 understand the degradation kinetics of the PPCP population markers, both linear regression (zero

162 order) and pseudo first order regression were fitted to the data obtained from the batch tests (Table

163 1). For the pseudo first order, the regression intercept was set through $100 \%$ at time 0 . The kinetic

164 model chosen for the degradation of each compound was based on the fit to the model, i.e. the

165 model with the higher $R^{2}$ value. For compounds where degradation observed was within the

166 uncertainty of measurements, we concluded that no degradation had occurred over the studied

167 period (12 hours) and thus these compounds are stable. The term "degradation" in this study refers

168 to either biological or chemical transformation as sorption to organic matter of the studied

169 chemicals was considered negligible as sorption is only considered significant for log octanol-water

170 partitioning coefficients (log $\mathrm{K}_{\mathrm{ow}}$ ) higher than $4.0^{30}$ (see SI Table 2 for log $\mathrm{K}_{\mathrm{ow}}$ values).

171 To our knowledge only Jelic et al. ${ }^{15}$ have investigated the fate of selected PPCPs under sewer

172 conditions. That study however was conducted only in a pressurised sewer which is similar to that of

173 the RM in our study. It is important to note though that the sewer reactors used for our experiment

174 had fixed area to volume $(A / V)$ ratios of $70.9 \mathrm{~m}^{2} / \mathrm{m}^{3}$ which is similar to $A / V$ ratios of small pipes but

175 higher than that of average sewers which typically use larger diameter pipes. Therefore the

176 chemicals investigated in this study may have had more contact with biofilms and hence more

177 degradation than would be expected in a typical sewer. This may explain why some discrepancy was

178 observed between our results and that of Jelic et al. ${ }^{15}$ who had an $A / V$ ratio of $8 \mathrm{~m}^{2} / \mathrm{m}^{3}$. As no other

179 studies have looked at the degradation within a GS, we conducted a comparison between our results

180 with available data on the removal efficiency of WWTPs for those PPCPs (Table 1). Such a

181 comparison helps support our findings although we acknowledge that fate and transport processes

182 during wastewater treatment are different from those during sewer passage ${ }^{31,32}$ (S1 SI). 
183 It was observed that there are two groups of chemicals, those which had no measurable degradation

184 (acesulfame, atenolol, carbamazepine, gabapentin and ibuprofen) and those which degraded over

185 the studied period with half-life estimates ranging from 0.6 to $10 \mathrm{~h}$ in the GS and 0.6 to $11 \mathrm{~h}$ in the

186 RM. Detailed discussion for the degradation of each chemical can be found in the SI.

187 

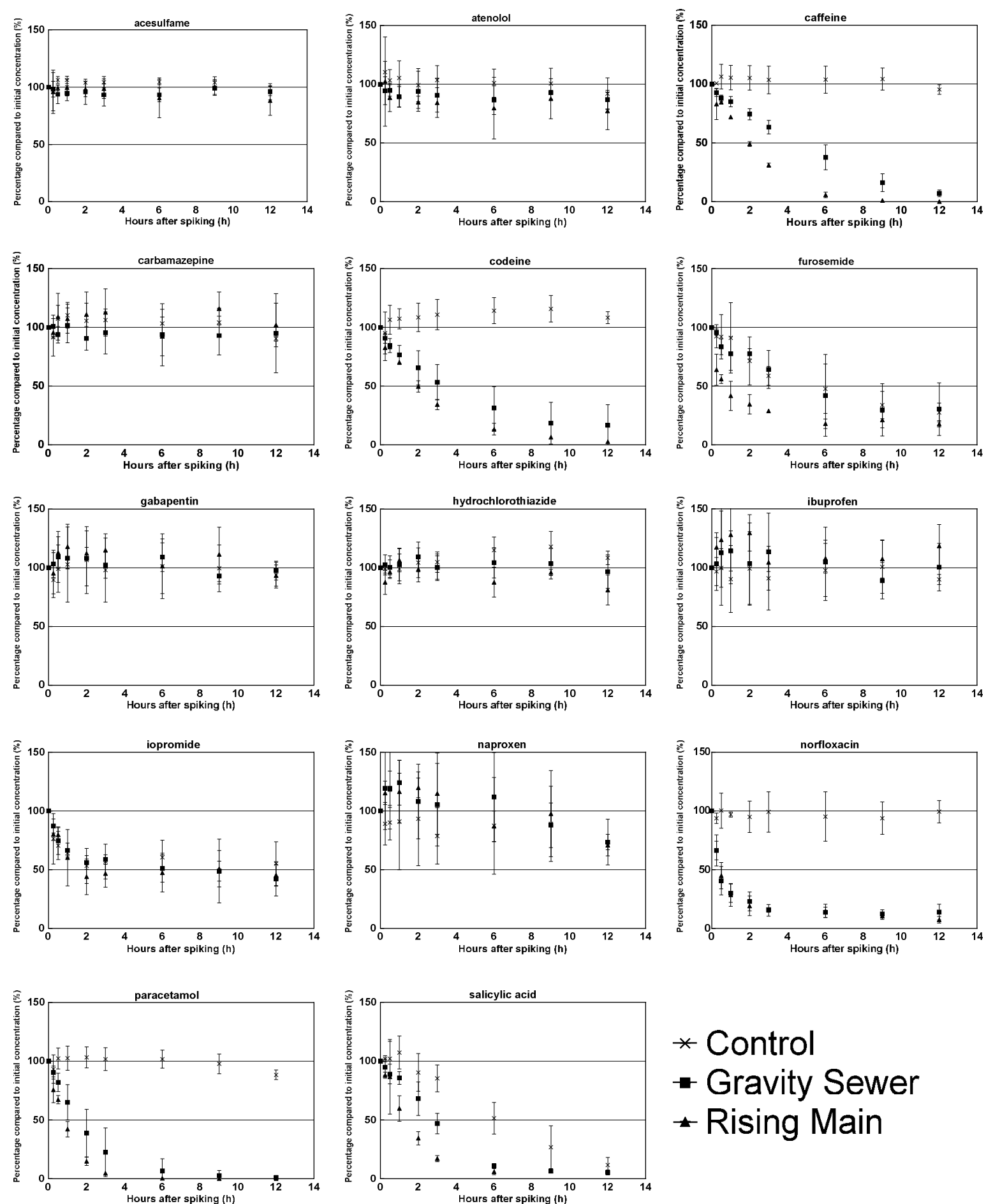

\section{* Control \\ - Gravity Sewer - Rising Main}

Figure 1 Degradation profiles of 14 population markers under different sewer conditions. Error bars represent the standard deviation of 3 replicate samples. The $x$ axis is the time after the feeding event. The $y$ axis is the concentration relative to the starting concentration (time zero) expressed as percent. Concentrations under control conditions are indicated by $\times$. Concentrations under gravity sewer (GS) conditions are indicated by $\mathbf{m}$. Concentrations under rising main (RM) conditions are indicated by $\boldsymbol{\Lambda}$. 


\section{Environmental Science \& Technology}

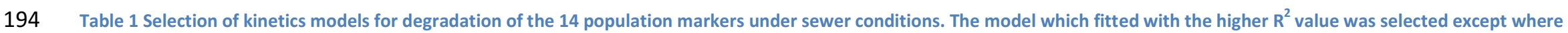

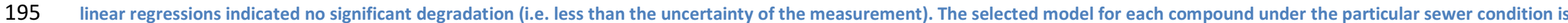

196 indicated by bolded text. Time before $10 \%$ loss under each sewer condition was calculated using the selected model for each compound. 


\begin{tabular}{|c|c|c|c|c|c|c|c|c|c|c|c|c|c|c|c|c|c|c|}
\hline & \multicolumn{2}{|c|}{$\begin{array}{l}\text { between mass } \\
\text { load and } \\
\text { population size }\end{array}$} & \multicolumn{2}{|c|}{ Control } & \multicolumn{2}{|c|}{ Gravity Sewer } & \multicolumn{2}{|c|}{ Rising Main } & \multicolumn{2}{|c|}{ Control } & \multicolumn{2}{|c|}{ Gravity Sewer } & \multicolumn{2}{|c|}{ Rising Main } & \multirow{2}{*}{ Control } & \multirow{2}{*}{$\begin{array}{l}\text { Gravity } \\
\text { Sewer }\end{array}$} & \multirow{2}{*}{$\begin{array}{l}\text { Rising } \\
\text { Main }\end{array}$} & \multirow[t]{2}{*}{$\begin{array}{c}\text { Removal } \\
\text { Efficiency \% }\end{array}$} \\
\hline & $R^{2}$ & Ranking $^{\mathrm{a}}$ & $\begin{array}{l}\text { Slope } \\
(\% / h)\end{array}$ & $R^{2}$ & $\begin{array}{l}\text { Slope } \\
\text { (\%/h) }\end{array}$ & $R^{2}$ & $\begin{array}{l}\text { Slope } \\
(\% / \mathrm{h})\end{array}$ & $R^{2}$ & $\begin{array}{l}\text { Half-life } \\
\text { (h) }\end{array}$ & $R^{2}$ & $\begin{array}{l}\text { Half-life } \\
\text { (h) }\end{array}$ & $R^{2}$ & $\begin{array}{l}\text { Half-life } \\
\text { (h) }\end{array}$ & $R^{2}$ & & & & \\
\hline Acesulfame & 0.995 & 1 & n.s. & & n.s. & & n.s. & & $>500$ & 0.005 & $>500$ & 0.000 & 100 & 0.075 & $>24$ & $>24$ & $>24$ & -32 \\
\hline Atenolol & 0.823 & 12 & n.s. & & n.s. & & n.s. & & 94 & 0.070 & 96 & 0.062 & 43 & 0.108 & $>24$ & $>24$ & $>24$ & $<0-85.1 \%^{15,33}$ \\
\hline Caffeine & 0.869 & 9 & n.s. & & $\begin{array}{l}-8.08 \\
\pm 0.37 \\
\end{array}$ & 0.952 & $\begin{array}{l}-8.27 \\
\pm 0.8 \\
\end{array}$ & 0.809 & 200 & 0.024 & 4.0 & 0.967 & 1.8 & 0.981 & $>24$ & 0.61 & 0.28 & $\begin{array}{c}>49.9- \\
99.6 \%^{31,33}\end{array}$ \\
\hline carbamazepine & 0.849 & 10 & n.s. & & n.s. & & n.s. & & 150 & 0.024 & 170 & 0.011 & $>500$ & 0.000 & $>24$ & $>24$ & $>24$ & $\begin{array}{c}<0- \\
62.3 \%^{15,31,34,35,} \\
{ }_{33}\end{array}$ \\
\hline Codeine & 0.908 & 8 & $\begin{array}{c}1.02 \\
\pm 0.49 \\
\end{array}$ & 0.117 & $\begin{array}{l}-7.13 \\
\pm 0.65 \\
\end{array}$ & 0.784 & $\begin{array}{l}-7.77 \\
\pm 0.73 \\
\end{array}$ & 0.820 & $>500$ & 0.000 & 3.8 & 0.867 & 2.1 & 0.983 & $>24$ & 0.57 & 0.32 & $13 \%^{35}$ \\
\hline Furosemide & 0.839 & 11 & $\begin{array}{l}-6.08 \\
\pm 0.77 \\
\end{array}$ & 0.715 & $\begin{array}{c}-5.9 \\
\pm 0.81 \\
\end{array}$ & 0.680 & $\begin{array}{r}-4.57 \\
\pm 0.91 \\
\end{array}$ & 0.501 & 5.7 & 0.764 & 5.8 & 0.731 & 1.9 & 0.708 & 0.87 & 0.88 & 0.29 & $40-80 \%^{36}$ \\
\hline Gabapentin & 0.968 & 2 & n.s. & & n.s. & & n.s. & & 460 & 0.001 & 90 & 0.062 & 110 & 0.029 & $>24$ & $>24$ & $>24$ & $\begin{array}{c}0-84 \%^{36} \\
6.4 \%^{37}\end{array}$ \\
\hline $\begin{array}{l}\text { hydrochloro- } \\
\text { thiazide }\end{array}$ & 0.944 & 3 & $\begin{array}{c}1.39 \\
\pm 0.42 \\
\end{array}$ & 0.252 & n.s. & & $\begin{array}{l}-1.11 \\
\pm 0.48 \\
\end{array}$ & 0.178 & $>500$ & 0.000 & 300 & 0.009 & 59 & 0.175 & $>24$ & $>24$ & 9.01 & $\begin{array}{l}0^{38,39}- \\
85 \%{ }^{40}\end{array}$ \\
\hline Ibuprofen & 0.919 & 6 & n.s. & & n.s. & & n.s. & & 320 & 0.002 & 71 & 0.029 & 270 & 0.004 & $>24$ & $>24$ & $>24$ & $72-100 \%^{36,33}$ \\
\hline lopromide & 0.377 & 14 & $\begin{array}{l}-2.26 \\
\pm 0.86 \\
\end{array}$ & 0.215 & $\begin{array}{l}-3.55 \\
\pm 0.63 \\
\end{array}$ & 0.557 & $\begin{array}{l}-2.99 \\
\pm 0.9 \\
\end{array}$ & 0.307 & 17 & 0.234 & 10 & 0.609 & 11 & 0.354 & 2.55 & 1.52 & 1.63 & $>80 \%{ }^{41}$ \\
\hline Naproxen & 0.912 & 7 & n.s. & & $\begin{array}{l}-3.15 \\
\pm 1.1 \\
\end{array}$ & 0.200 & $\begin{array}{c}-3.26 \\
\pm 0.94 \\
\end{array}$ & 0.323 & 48 & 0.038 & 23 & 0.193 & 22 & 0.314 & $>24$ & 3.17 & 3.07 & $\begin{array}{c}43.3- \\
98.6 \%^{34,33}\end{array}$ \\
\hline Norfloxacin & 0.929 & 4 & n.s. & & $\begin{array}{r}-4.46 \\
\pm 1.1 \\
\end{array}$ & 0.396 & $\begin{array}{r}-4.89 \\
\pm 1.11 \\
\end{array}$ & 0.439 & $>500$ & 0.001 & 0.6 & 0.819 & 0.6 & 0.864 & $>24$ & 0.09 & 0.09 & $\begin{array}{c}87- \\
100 \%^{31,42,43} \\
\end{array}$ \\
\hline paracetamol & 0.811 & 13 & n.s. & & $\begin{array}{c}-8.31 \\
\pm 0.96\end{array}$ & 0.693 & $\begin{array}{l}-6.74 \\
\pm 1.2\end{array}$ & 0.560 & 120 & 0.070 & 1.5 & 0.949 & 0.8 & 0.986 & $>24$ & 0.22 & 0.12 & $98.7-100 \% \%^{44,33}$ \\
\hline salicylic acid & 0.922 & 5 & $\begin{array}{l}-8.26 \\
\pm 0.55 \\
\end{array}$ & 0.899 & $\begin{array}{l}-8.79 \\
\pm 0.66 \\
\end{array}$ & 0.850 & $\begin{array}{c}-7.49 \\
\pm 1.14 \\
\end{array}$ & 0.635 & 5.2 & 0.869 & 2.6 & 0.953 & 1.3 & 0.929 & 1.21 & 0.40 & 0.20 & $\begin{array}{c}89.6- \\
100 \%^{42,45,33}\end{array}$ \\
\hline
\end{tabular}

198 
1993.3 Implications of in-sewer degradation for PPCPs on a population model and recommendations

200 for future population models

201 The sewer degradation data obtained from this study represents a worst case scenario in terms of

202 degradation processes as the A/V ratio was $70.9 \mathrm{~m}^{2} / \mathrm{m}^{3}$ which is about 2-10 times higher than that of

203 average sewers. It can then be suggested that uncertainty from degradation is not an issue for

204 acesulfame, gabapentin, atenolol, carbamazepine and ibuprofen as these compounds showed no

205 measurable degradation under any sewer conditions over the 12 hour study period. Their stability

206 probably help acesulfame and gabapentin achieve the first and second ranking in our list of potential

207 population markers with high correlation between mass load and population size. The linear

208 regressions also take into account uncertainties such as degradation/transformation in the sewer,

209 variability from the different sampling methods, chemical analysis, and any differences in

210 consumption and excretion in the different regions. This is because the samples collected in our

211 earlier study were from sewer catchments of both small and large population sizes and from

212 different localities and jurisdictions within Australia - using different sampling modes and

213 frequencies. Therefore, it is likely that the variability observed in the linear regressions for the mass

214 loads of these population markers versus population size presented in O'Brien et al. ${ }^{13}$ reflects the

215 variance associated with other factors such as differences in consumption and excretion as well as

216 uncertainty associated with sampling and analysis.

217 To understand if there is a link between degradation and the correlations between mass loads and

218 population, we correlated the $\mathrm{R}^{2}$ values for the correlation between mass load and population size

219 from the previous paper ${ }^{13}$ and the time taken for $10 \%$ loss under each of the sewer conditions in

220 this study and found that there was no relationship (Spearman's rank correlation, $p<0.05)(\mathrm{SI}$ Table

221 3). For example, norfloxacin was considered unstable under sewer conditions but had better

222 correlation $\left(R^{2}=0.912\right)$ between population size and mass load than carbamazepine $\left(R^{2}=0.849\right)$

223 which was stable under all sewer conditions. One explanation for why degradation did not affect the 
correlations between mass loads and population is that for degradation to affect a correlation there must be a crossover between residence time and degradation (e.g. the observed degradation in some catchments would be higher than others because of longer residence times). We speculate that other factors may be responsible for the higher uncertainty but this requires further investigation.

To better understand the impact of in-sewer degradation on the uncertainty of our population estimate, we compared the coefficient of variation (CV) of the population estimate for each catchment based on all chemicals (s14 in the paper); the stable chemicals identified in this study and the unstable chemicals identified in this study (Figure 2). Individual substance distributions for the three largest WWTPs are shown in SI Figure 2. Comparing stable chemicals with all chemicals (stable and unstable chemicals combined), for all WWTP catchment population estimates made using the model, the CV was lower (higher precision) by using all 14 chemicals in the model. However, comparing stable chemicals with unstable chemicals a few observations can be made. Firstly, the CV decreases (precision increases) as population increases regardless of the chemicals used in the model. For unstable chemicals however, the uncertainty appears to plateau at $\sim 20 \%$. Secondly, we observe across all catchment sizes, stable chemicals produce more precise estimates than unstable chemicals and that the difference in the CV increases as catchment size increases. The model used a constant error term which means the magnitude in the deviation in mass loads is constant across population sizes (i.e. mass/day/person uncertainty stays constant across populations). It would then be expected that smaller differences would be observed between unstable and stable chemicals with smaller population sizes, but as the population increases, the difference in precision also increases. Despite this, the third observation is that the inclusion of stable and unstable chemicals in the model resulted in the most precise estimates. In Bayesian inference, the posterior distribution is the product of the likelihood function and prior probability. In our model, the chemicals with the highest correlation between mass load and population size (as indicated by their R2 value) implicitly have the most weight on the likelihood that a given estimate will provide accurate population 

meant that the less stable chemicals had less impact on the posterior distribution.

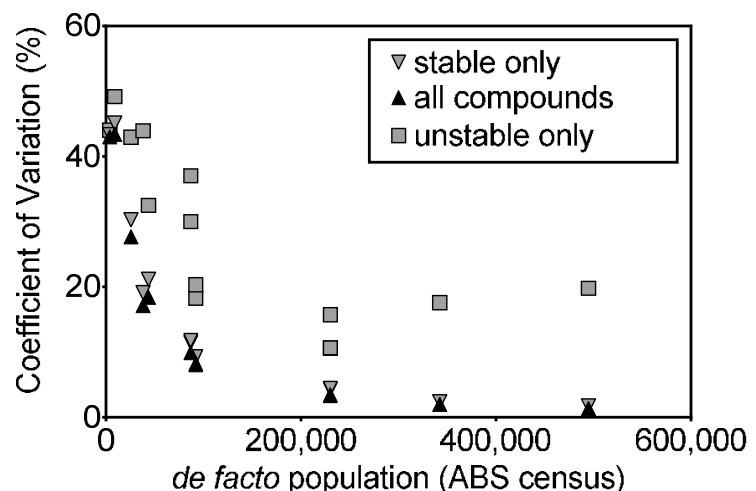

Figure 2 Coefficient of variation expressed as percentage for posterior estimates for population size for each of the WWTPs from O'Brien et al. (2014) using all chemicals (s14, indicated by $\mathbf{\Delta}$ ), using only the chemicals identified as stable in the sewer by this study (indicated by $\nabla$ ) and using only the chemicals identified as unstable in the sewer by this study (indicated by $\mathbf{0}$ ).

257 For future calibration of a population model, if the only chemicals identified are unstable in the sewer and/or where the stable chemicals have no relationship between mass load and population size, we propose to still use less stable markers but also include a metabolite/metabolites or transformation products which are produced from the degradation of the compound within the sewer. This will then provide insight into degradation within the sewer and to also counterbalance the impact of the degradation of the parent chemical on the model i.e. as the mass load of the parent chemical decreases from in-sewer activity, the mass load of the metabolites/transformation products increases. Additionally, as shown in the earlier study, the inclusion of more chemicals (even those with lower correlation) in the model lead to increased precision of the population estimate.

\subsection{Importance of the quality of calibration data used in a population model}

The samples from our previous study used to calibrate the model covered a broad range of catchments from across Australia including small catchments, large catchments, different climate and geographic zones (from cold temperate to subtropical), inland and coastal catchments and catchments with potential cultural and socioeconomic differences. These factors may all influence 
271 the consumption, excretion and degradation of the population markers investigated and thus

272 influence the calibration data. Despite these factors, the correlation between mass load and

273 population size was still above $\mathrm{R}^{2}=0.8$ for 13 of the 14 population markers.

274 In the model, an error term was included that accounts for differences in consumption between

275 locations and daily variations of population size. It was implied that daily variations would include

276 factors that would contribute to either the addition (increase in mass load) or removal (decrease in

277 mass load) of a chemical in wastewater.

278 Degradation is therefore one variable in daily variations and its effect on the population model is the

279 extent that the degradation of a chemical would affect the variance in the correlation between mass

280 load and the population size. However, if the combined impact of $A / V$ ratios and mean residence

281 times are all similar among catchments, then the chemicals would degrade to a similar extent and

282 we would get a systematic underestimation of total consumption. In such a case, there is still a good

283 correlation between mass load and population size (assuming relatively homogenous consumption

284 and excretion) and thus we expect that the population estimate would be unaffected by the

285 degradation. The quality of the population estimates is therefore dependent on the quality

286 (representativeness) of the calibration data and as such, calibration data for a different area or

287 country should include catchments of both long and short residence times as well as a combination

288 of GS and RM sewers. By including these in the calibration data, variability due to degradation is

289 covered implicitly. Using in-situ calibration data, the $\mathrm{R}^{2}$ term helps explain homogeneity in

290 consumption of all chemicals across catchments. To highlight this, the two highest $\mathrm{R}^{2}$ correlations

291 between mass load and population size were acesulfame and gabapentin. Acesulfame is an artificial

292 sweetener, gabapentin is a medication used to treat epilepsy, neuropathic pain and a range of other

293 conditions. Despite the difference in intended consumption, in order for them to have such high $\mathrm{R}^{2}$

294 correlations there needed to be homogenous consumption amongst all catchments in the calibration

295 dataset. Assuming non-homogenous consumption, the $\mathrm{R}^{2}$ values would be lower and their influence

296 on the model would diminish empirically. 
Degradation within sewers is dependent on the residence time in the sewer. Using data from the most comprehensive WBE study which covered 25 WWTPs in 2012 and 47 WWTPs in $2013^{4}$, we correlated mean residence time of each WWTP who provided a mean residence time $(n=50)$ against population size (Spearman's rank correlation, $\rho<0.05$ ) and found there is no relationship between mean residence time and population size (SI Figure 1). Therefore we consider that residence time is independent of population size and as such degradation can also be considered independent of population size.

In our previous study ${ }^{13}$, chemicals for population estimation were chosen using two criteria: (1) the chemical must be measurable via direct injection on LC-MSMS in all of the collected samples; (2) the mass load of the chemical must show a correlation with population size $\left(R^{2}>0.8\right)$. With the use of Bayesian inference to combine the chemicals which met these criteria into a population model it was apparent that they were capable of estimating the population size with high accuracy and that uncertainty reduced with the inclusion of more markers. While these two criteria were observed as suitable criteria for our specific catchments, to extend the applicability of the population model to any other catchments based on the knowledge about chemical degradation obtained in this study we now propose two additional criteria for "Best Practice" population markers: (3) degradation should be negligible under CR conditions to ensure the stability of chemicals during wastewater collection; and (4) the mean residence time of the WWTP sewer network (RM and/or GS) should be shorter than the time for $10 \%$ degradation to occur for the compound under sewer conditions.

317 Degradation of less than $10 \%$ is considered acceptable for our population markers as it is the acceptable "Best Practice" tolerance for degradation of sewage drug biomarkers ${ }^{1}$. If no mean residence time is provided by WWTPs, we recommend using the $95^{\text {th }}$ percentile of the mean residence time of all WWTPs (non-normally distributed) from the Ort et al. ${ }^{4}$ study which was 10 
321 hours. A protocol for selecting "Best Practice" suitable population markers based on these criteria is 322 shown in Figure 3.

323 Based on these criteria, for our calibration data and catchments, acesulfame, atenolol, carbamazepine, gabapentin and ibuprofen would all be considered "Best Practice" population markers particularly considering they had negligible degradation under "high in-sewer degradation processes" and hence should be considered as potential population markers for all sites regardless of knowledge of mean residence time. Their stability was also greater than the longest mean residence time of the sewer catchments in the Ort et al. ${ }^{4}$ study (15 hours). Hydrochlorothiazide can also be considered as a potential "Best Practice" population marker providing the sewer catchments do not have rising main sewers with mean residence times greater than 9 hours (Table 1).

Furosemide, iopromide and salicylic acid were identified as highly degradable as all compounds degraded in the CR and thus should not be used as "Best Practice" marker (failing criteria 3). The use of naproxen, caffeine, codeine, norfloxacin and paracetamol as "Best Practice" markers would require further investigation as all had greater than $10 \%$ loss in $<3$ hours under both gravity and rising main sewer conditions (Table 1).

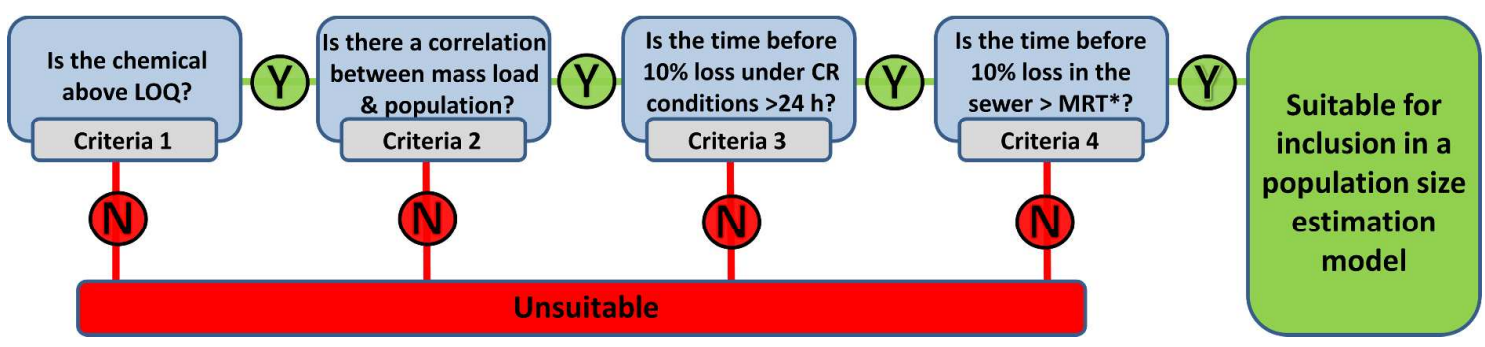

Figure 3 Selection criteria of "Best Practice" population markers based on measurable levels (i.e. above LOQ), degradation in wastewater and within the sewer and correlation between mass load and population. $\mathrm{Y}=\mathrm{Yes}$. $\mathrm{N}=\mathrm{No}$. $\mathrm{LOQ}=$ limit of quantification. MRT = mean residence time. ${ }^{*}=$ if mean residence time is unknown, assume $10 \mathrm{~h}$ as it is the $95^{\text {th }}$ percentile of the mean residence times from the largest wastewater-based epidemiology study ${ }^{4}$.

\section{Supporting Information}

342 Comparison of sewer degradation of the population markers in this study against the literature. LCMS/MS parameters. Population marker log $\mathrm{K}_{\mathrm{ow}}$ values. Pearson correlation analysis between the $\mathrm{R}^{2}$ for the correlation between mass load and population size and time before $10 \%$ loss in the sewer 
reactors. Mean residence time versus population size. Individual substance distributions for

estimating population size for all Large WWTPs.

\section{Acknowledgements}

The authors would like to thank Andreas Scheidegger from Eawag for his invaluable assistance in the

initial model development and for the advice he provided for this study. The Queensland Alliance

for Environmental Health Sciences, The University of Queensland, gratefully acknowledges the

financial support of the Queensland Department of Health. This project was conducted as part of an

ARC Discovery project (DP150100645). Jake O'Brien and Andrew Novic are both funded by APA PhD

Scholarships. Phong K. Thai is funded by the QUT Vice Chancellor Research Fellowship. Jochen

Mueller is funded through the ARC Future Fellowship (FT120100546). Guangming Jiang is funded by

a Queensland State Government Early Career Accelerate Fellowship.

1. Castiglioni, S.; Bijlsma, L.; Covaci, A.; Emke, E.; Hernandez, F.; Reid, M.; Ort, C.; Thomas, K. V.; van Nuijs, A. L. N.; de Voogt, P.; Zuccato, E., Evaluation of Uncertainties Associated with the Determination of Community Drug Use through the Measurement of Sewage Drug Biomarkers. Environ. Sci. Technol. 2013, 47, (3), 1452-1460. O'Brien, J. W.; Prichard, J.; Thai, P. K.; Ort, C., Systematic and Day-to-Day Effects of Chemical-Derived Population Estimates on Wastewater-Based Drug Epidemiology. Environ Sci Technol 2015, 49, (2), 10.

3. Lai, F. Y.; Ort, C.; Gartner, C.; Carter, S.; Prichard, J.; Kirkbride, P.; Bruno, R.; Hall, W.; Eaglesham, G.; Mueller, J. F., Refining the estimation of illicit drug consumptions from wastewater analysis: Co-analysis of prescription pharmaceuticals and uncertainty assessment. Water Research 2011, 45, (15), 4437-4448.

4. Ort, C.; van Nuijs, A. L.; Berset, J. D.; Bijlsma, L.; Castiglioni, S.; Covaci, A.; de Voogt, P.; Emke, E.; Fatta-Kassinos, D.; Griffiths, P.; Hernandez, F.; Gonzalez-Marino, I.; Grabic, R.; Kasprzyk-Hordern, B.; Mastroianni, N.; Meierjohann, A.; Nefau, T.; Ostman, M.; Pico, Y.; Racamonde, I.; Reid, M.; Slobodnik, J.; Terzic, S.; Thomaidis, N.; Thomas, K. V., Spatial differences and temporal changes in illicit drug use in Europe quantified by wastewater analysis. Addiction 2014, 109, (8), 1338-52. 5. van Nuijs, A. L. N.; Mougel, J. F.; Tarcomnicu, I.; Bervoets, L.; Blust, R.; Jorens, P. G.; Neels, H.; Covaci, A., A one year investigation of the occurrence of illicit drugs in wastewater from Brussels, Belgium. J. Environ. Monit. 2011, 13, (4), 1008-1016. 
378 6. Baker, D. R.; Barron, L.; Kasprzyk-Hordern, B., Illicit and pharmaceutical drug consumption 379 estimated via wastewater analysis. Part A: chemical analysis and drug use estimates. Sci Total 380 Environ 2014, 487, 629-41.

381 7. Banta-Green, C. J.; Field, J. A.; Chiaia, A. C.; Sudakin, D. L.; Power, L.; De Montigny, L., The 382 spatial epidemiology of cocaine, methamphetamine and 3,4-methylenedioxymethamphetamine 383 (MDMA) use: a demonstration using a population measure of community drug load derived from 384 municipal wastewater. Addiction 2009, 104, (11), 1874-1880.

385 8. Burgard, D. A.; Banta-Green, C.; Field, J. A., Working upstream: how far can you go with 386 sewage-based drug epidemiology? Environ. Sci. Technol. 2014, 48, (3), 1362-8.

387 9. Been, F.; Rossi, L.; Ort, C.; Rudaz, S.; Delemont, O.; Esseiva, P., Population normalization with 388 ammonium in wastewater-based epidemiology: application to illicit drug monitoring. Environ Sci 
428 24. OECD, Test No. 314: Simulation Tests to Assess the Biodegradability of Chemicals Discharged 429 in Wastewater. OECD Publishing: 2008.

$430 \quad 25 . \quad$ Plosz, B. G.; Reid, M. J.; Borup, M.; Langford, K. H.; Thomas, K. V., Biotransformation kinetics 431 and sorption of cocaine and its metabolites and the factors influencing their estimation in 432 wastewater. Water Res 2013, 47, (7), 2129-40.

433 26. van Nuijs, A. L. N.; Abdellati, K.; Bervoets, L.; Blust, R.; Jorens, P. G.; Neels, H.; Covaci, A., The 434 stability of illicit drugs and metabolites in wastewater, an important issue for sewage epidemiology? 435 J. Hazard. Mater. 2012, 239-240, 19-23.

$43627 . \quad$ Chen, C.; Kostakis, C.; Irvine, R. J.; Felgate, P. D.; White, J. M., Evaluation of pre-analysis loss 437 of dependent drugs in wastewater: stability and binding assessments. Drug Testing and Analysis $438 \quad 2012,5,(8), 6$.

439 28. Guisasola, A.; de Haas, D.; Keller, J.; Yuan, Z., Methane formation in sewer systems. Water 440 Res 2008, 42, (6-7), 1421-30.

$44129 . \quad$ Jiang, G.; Gutierrez, O.; Yuan, Z., The strong biocidal effect of free nitrous acid on anaerobic 442 sewer biofilms. Water Research 2011, 45, (12), 3735-3743.

443 30. Thompson, K.; Zhang, J.; Zhang, C., Use of fugacity model to analyze temperature-dependent 444 removal of micro-contaminants in sewage treatment plants. Chemosphere 2011, 84, (8), 1066-71.

445 31. Liu, J. L.; Wong, M. H., Pharmaceuticals and personal care products (PPCPs): a review on 446 environmental contamination in China. Environ Int 2013, 59, 208-24.

447 32. Blair, B.; Nikolaus, A.; Hedman, C.; Klaper, R.; Grundl, T., Evaluating the degradation, 448 sorption, and negative mass balances of pharmaceuticals and personal care products during 449 wastewater treatment. Chemosphere 2015, 134, 395-401.

450 


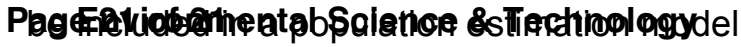

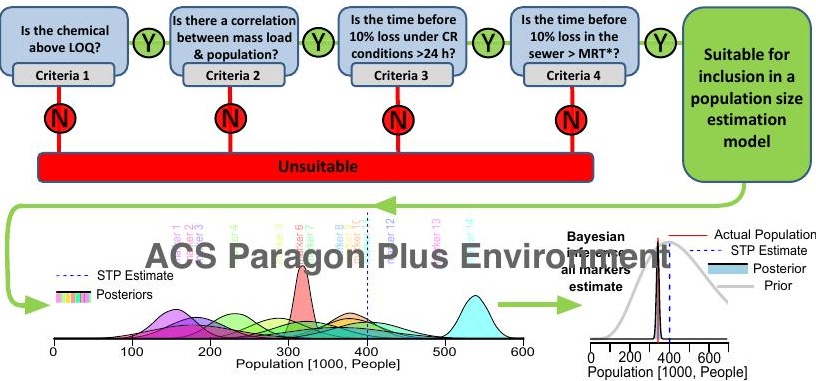

